\title{
Tunelizador maleável moldável universal na cirurgia de revascularização do membro inferior isquêmico
}

\author{
Soft moldable universal tunneler in bypass surgery \\ for lower limb ischemia
}

Fabio Henrique Rossi ${ }^{1}$, Nilo Mitsuru Izukawa ${ }^{1}$, Lannes Alberto Oliveira ${ }^{1}$, Wilson Trópico ${ }^{1}$,
José Nicodemos Tobias ${ }^{2}$, Akash Kuzhiparambil Prakasan ${ }^{1}$, Heraldo Antônio Barbato ${ }^{1}$

\section{Resumo}

Contexto: A revascularização do membro inferior isquêmico envolve a criação de túnel para a acomodação do enxerto vascular cujo trajeto e extensão dependem do local da anastomose proximal e distal do enxerto. $\mathrm{Na}$ atualidade, os tunelizadores utilizados consistem em estruturas cilíndricas metálicas rígidas.

Objetivo: Testar a utilização de um tunelizador universal metálico e moldável na revascularização de membros inferiores isquêmicos.

Métodos: O tunelizador desenvolvido consiste de uma estrutura cilíndrica híbrida e modular composta de fio de aço inoxidável moldável interno, com manopla em extremidade proximal, dupla ponta cônica intercambiável em extremidade distal e bainha cilíndrica externa de polietileno. O tunelizador foi utilizado em cirurgias de revascularização do membro inferior isquêmico, independentemente do tipo, trajeto e extensão do enxerto escolhido.

Resultados: As características de maleabilidade e capacidade de deformação permitiram a adaptação e utilização do mesmo tunelizador nos diversos tipos de enxerto realizados. A ponta cônica distal com duplo diâmetro, intercambiável, permitiu que o mesmo aparato pudesse ser utilizado na cirurgia de revascularização com veia safena reversa ou prótese sintética

Conclusão: $\mathrm{O}$ tunelizador metálico maleável moldável pode ser utilizado com segurança e apresenta vantagens nas cirurgias de revascularização dos membros inferiores isquêmicos e possivelmente em outros territórios vasculares isquêmicos. Sua versatilidade e baixo custo de produção podem levá-lo a substituir os tunelizadores rígidos utilizados na atualidade.

Palavras-chave: Enxerto, membro inferior, ponte, isquemia.

\section{Introdução}

A cirurgia de revascularização do membro inferior isquêmico envolve a criação de um túnel para a acomodação do enxerto vascular, seja ele realizado com a veia safena magna reversa ou com prótese sintética. O trajeto e a extensão do túnel dependem da posição do segmento arterial escolhido para a realização da anastomose proximal e distal do enxerto. A confecção desses túneis e a passagem do

\begin{abstract}
Background: Bypass surgery for lower limb ischemia involves the creation of a tunnel for accommodating the vascular graft. Tunnel shape and length depend on the site chosen for proximal and distal anastomosis. Tunnelers currently employed are rigid metallic cylindrical structures.
\end{abstract}

Objective: To test the use of a moldable universal metallic tunneler in bypass surgeries for lower limb ischemia.

Methods: The assessed tunneler is a hybrid, modular, cylindrical structure made of moldable stainless steel wire, with a handle on the proximal end, a tapered distal tip with two interchangeable diameter options on the distal end, and an outer cylindrical polyethylene sheath. The same tunneler was used in all bypass surgeries for lower limb ischemia, regardless of type, shape, and length of the graft chosen.

Results: Tunneler moldability and deformation capacity made it possible to adapt and use the same device in combination with several different types of grafts. The tapered distal tip with two diameter options allowed the tunneler to be used in surgeries performed with both reversed saphenous vein and synthetic graft.

Conclusion: The soft moldable universal tunneler tested in this study can be used safely and proved beneficial in bypass surgeries for lower limb ischemia. The versatility and low production cost involved may lead to replacement of the rigid tunnelers currently employed.

Keywords: Graft, lower limb, bypass, ischemia.

enxerto através dos mesmos muitas vezes são feitas às cegas. Muito provavelmente, apesar da escassa literatura sobre o tópico, desde os primórdios da cirurgia de restauração arterial o cirurgião preocupou-se com esse importante e delicado tempo cirúrgico e idealizou as mais diversas técnicas e instrumentos. Na atualidade, os tunelizadores utilizados consistem em estruturas cilíndricas metálicas rígidas, que podem limitar ou dificultar a confecção dos tú-

1. Departamento de Cardiologia, Instituto Dante Pazzanese de Cardiologia (IDPC), São Paulo, SP.

2. Departamento de Bioengenharia, IDPC, São Paulo, SP.

Não foram declarados conflitos de interesse associados à publicação deste artigo.

Artigo submetido em 02.10.09, aceito em 16.03.10.

$J$ Vasc Bras. 2010;9(1):21-24.

Copyright (C) 2010 by Sociedade Brasileira de Angiologia e de Cirurgia Vascular 
neis e até mesmo causar lesões iatrogênicas em estruturas ou órgãos presentes no trajeto do enxerto ${ }^{1,2}$. Neste estudo, tivemos como objetivo testar a utilização de um tunelizador universal metálico e moldável na cirurgia de revascularização de membros inferiores isquêmicos.

\section{Método}

O tunelizador metálico, maleável, moldável e universal desenvolvido no Laboratório de Bioengenharia do Instituto Dante Pazzanese de Cardiologia, São Paulo (SP), consiste de uma estrutura cilíndrica híbrida e modular composta de: 1) fio de aço inoxidável maleável e moldável interno, com manopla em extremidade proximal e dupla ponta cônica intercambiável em extremidade distal; 2) bainha cilíndrica externa de polietileno transparente (Figura 1).

O tunelizador foi utilizado em cirurgias de revascularização do membro inferior isquêmico no Setor de Cirur-

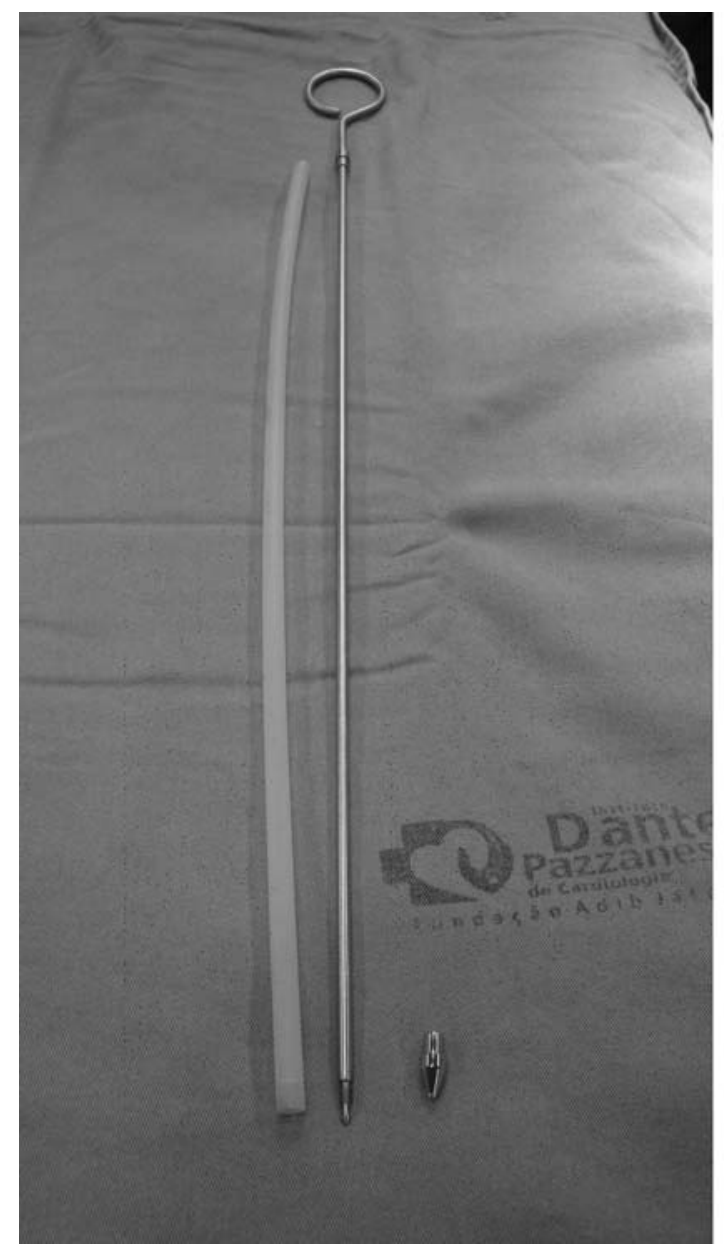

gia Vascular do Instituto Dante Pazzanese de Cardiologia. O mesmo aparato foi utilizado em todas as cirurgias realizadas, independentemente do tipo, trajeto e extensão do enxerto escolhido.

\section{Resultado}

A experiência inicial obtida com o tunelizador foi realizada nos tipos de enxertos de revascularização de membros inferiores isquêmicos descritos na Tabela 1 .

As características de maleabilidade e capacidade de deformação do tunelizador (Figura 2) permitiram sua adaptação e utilização nos diversos tipos de enxerto realizados. A ponta cônica distal com duplo diâmetro, intercambiável, permitiu que o mesmo aparato fosse utilizado na cirurgia de revascularização tanto com veia safena reversa quanto com prótese sintética.
A

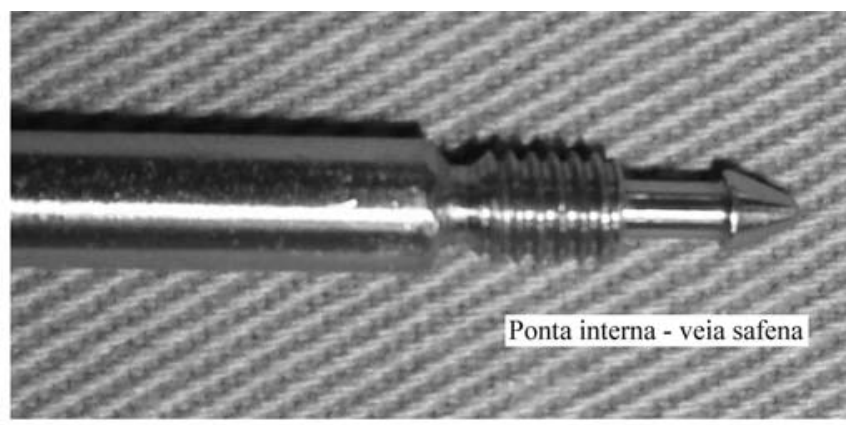

B
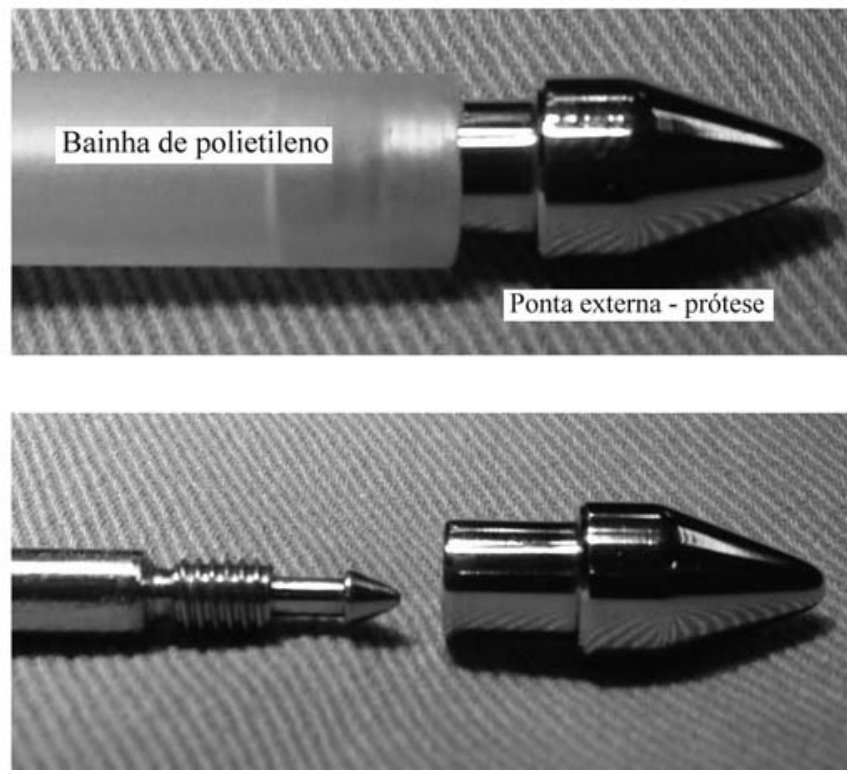

Figura 1 - Tunelizador metálico. A) Detalhe de ponta interna (tunelização da veia safena magna); B) ponta externa (tunelização de prótese) e bainha de polietileno 


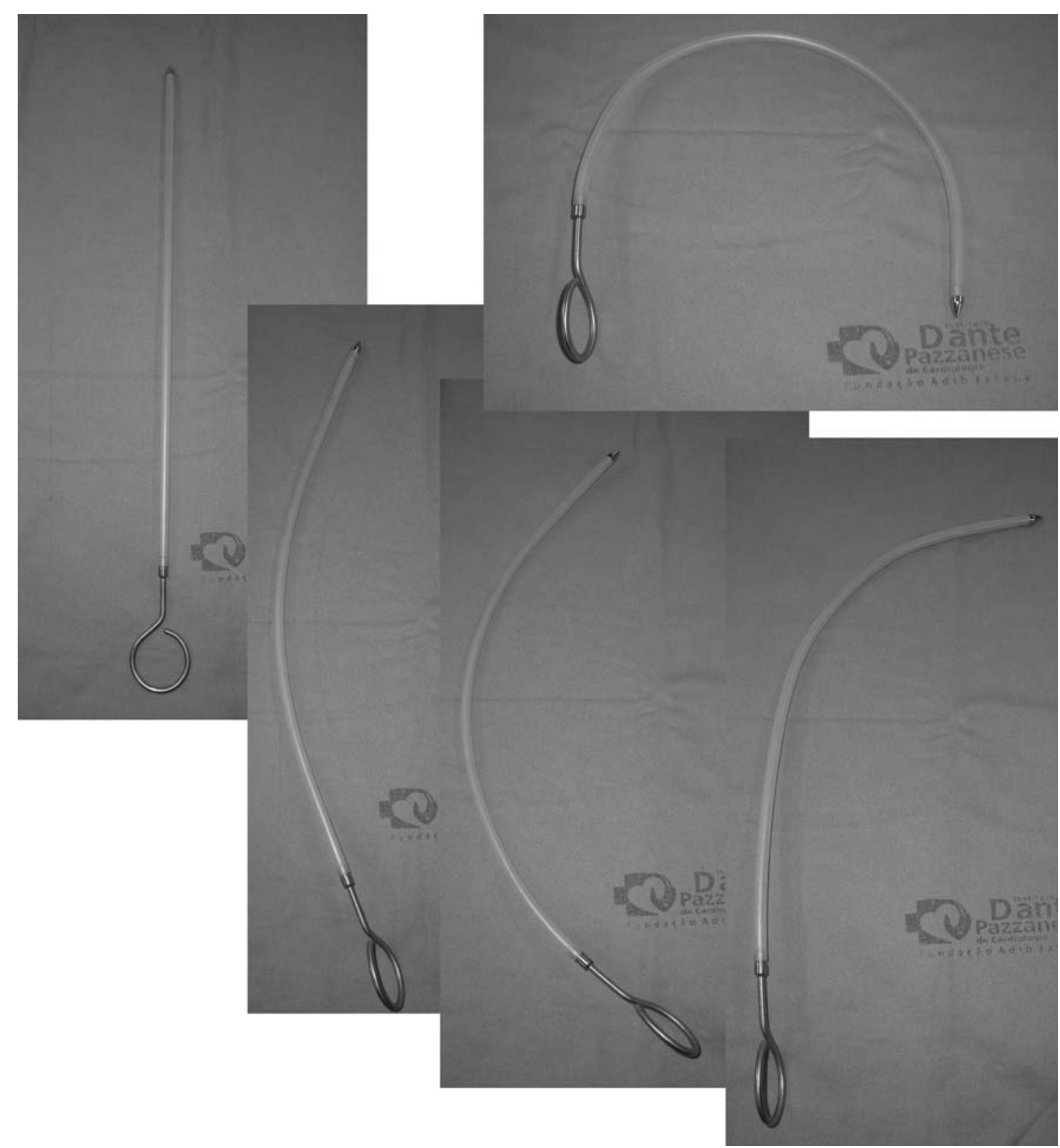

Figura 2 - Tunelizador metálico maleável moldável universal: o mesmo aparato pode assumir diversas angulações e ser utilizado em diferentes tipos de enxertos

Tabela 1 - Cirurgias de revascularização de membros inferiores isquêmicos realizadas com o tunelizador universal

\begin{tabular}{lccc}
\hline Idade do paciente (anos) & Indicação & Cirurgia & Complicação \\
\hline 74 & Lesão trófica & Enxerto fe-po supra VSMR & - \\
82 & Lesão trófica & Enxerto fe-ta VSMR & - \\
64 & Dor em repouso & Enxerto aorto-bifemoral & - \\
54 & Claudicação limitante & Enxerto áxilo-bifemoral & - \\
65 & Lesão trófica & Enxerto fêmoro-femoral cruzado & - \\
68 & Dor em repouso & Enxerto ilíaco-femoral & \\
\hline
\end{tabular}

VSMR = veia safena magna reversa; fe-po = fêmoro poplíteo; fe-ta = fêmoro tibial anterior. 
No caso da tunelização da veia safena magna reversa, a passagem do tunelizador entre a extremidade proximal e distal é realizada no interior da proteção da bainha de polietileno, evitando torção, estiramento e lesão da ligadura dos ramos colaterais laterais ou acotovelamentos.

\section{Discussão}

O enxerto autógeno ou sintético é comumente utilizado na isquemia dos membros inferiores. A falha técnica é a principal causa de obstrução precoce do enxerto, sendo a torção, a tração, o estiramento, o acotovelamento e o esgarçamento os principais mecanismos. Além do cuidado no momento da realização das anastomoses, a tunelização é uma etapa importantíssima para o sucesso da cirurgia ${ }^{3}$.

A utilização de tunelizadores rígidos pré-moldados em curvaturas predeterminadas pode limitar sua adaptação ou até mesmo causar lesões a estruturas e órgãos presentes em seu trajeto ${ }^{4}$. Além disso, sabemos que uma das complicações mais temidas na cirurgia de revascularização do membro isquêmico é a infecção da ferida operatória e do próprio enxerto. O número de incisões e dissecção cirúrgica necessários durante a tunelização do enxerto podem estar envolvidos nesse tipo de complicação. A utilização do equipamento aqui apresentado pode diminuir o número de incisões necessárias e, provavelmente, o índice de infecção cirúrgica.

A doença aterosclerótica obstrutiva é uma doença sistêmica que pode acometer diversos territórios arteriais. A variabilidade de extensão, gravidade e território acometido tornam infinitas as possibilidades de estratégias cirúrgicas necessárias, o que salienta a importância das características do tunelizador moldável e maleável aqui apresentado na cirurgia de revascularização dos membros inferiores isquêmicos.

As características do tunelizador testado podem tornar possível sua aplicação em outros territórios e tipos de cirurgia, tais como enxertos extra-anatômicos para os troncos supra-aórticos (enxerto carotídeo subclávio, carotí- deo-carotídeo, áxilo-axilar), derivações aórticas supradiafragmáticas para artérias viscerais e tunelização de via de acesso temporário ilíaco no tratamento endovascular das doenças da aorta, fístulas arteriovenosas, etc.

\section{Conclusão}

O tunelizador metálico maleável moldável pode ser utilizado com segurança e apresenta vantagens nas cirurgias de revascularização dos membros inferiores isquêmicos e possivelmente em outros territórios vasculares isquêmicos. Sua versatilidade e baixo custo de produção podem levá-lo a substituir os tunelizadores rígidos utilizados na atualidade.

\section{Referências}

1. Parsonnet V, Driller J. A tunneler for bypass vascular surgery. Arch Surg. 1973;106:236-7.

2. Blumenberg RM, Gelfand ML. A simple and inexpensive tunneler for use in peripheral vascular surgery. Surgery. 1974;75:305-7.

3. Owens CD, Ho KJ, Conte MS. Lower extremity vein graft failure: a translational approach. Vasc Med. 2008;13:63-74.

4. Mouton WG, Otten KT, Fitridge RA. Proximalized lateral tunnel for the bypass to the dorsalis pedis artery: a safe way to go. Thorac Cardiovasc Surg. 2001;49:245-6.

Correspondência:

Fabio Henrique Rossi

E-mail: vascular369@hotmail.com

\section{Contribuições dos autores}

Concepção e desenho do estudo: FHR, WT, JNT

Análise e interpretação dos dados: FHR

Coleta de dados: FHR

Redação do artigo: FHR

Revisão crítica do texto: FHR, NMI, LAO, HAB, AKP

Aprovação final do artigo*: FHR, NMI, LAO, WT, JNT, AKP, HAB

Análise estatística: N/A

Responsabilidade geral pelo estudo: FHR

Informações sobre financiamento: N/A

* Todos os autores leram e aprovaram a versão final submetida ao J Vasc Bras. 